\section{Effects of Lactate Dehydrogenase (LDH) in Preeclampsia.}

Ababio $\mathrm{GK}^{1^{*}}$, Adu-Bonsaffoh $\mathrm{K}^{2}$, Narh $\mathrm{G}^{3}$, Morvey $\mathrm{D}^{4}$, Botchway $\mathrm{F}^{5}$, Abindau $\mathrm{E}^{6}$, Neequaye $\mathrm{J}^{7}$ and Quaye $\mathrm{IKE}^{8}$

${ }^{1}$ Department of Medical Biochemistry, University of Ghana School of Biomedical and Allied Health Sciences, Legon, Accra, Ghana

${ }^{2}$ Department of Obstetrics and Gynaecology, Korle-Bu Teaching Hospital, Accra, Ghana

${ }^{3}$ Department of Biochemistry, University of Ghana, Accra, Ghana

${ }^{4}$ Clinical Documentation Improvement Specialist, Southern California Hospital, Culver City, California, USA

${ }^{5}$ Department of Child Health, Korle-Bu Teaching Hospital, Accra, Ghana

${ }^{6}$ Department of Physiology, University of Ghana SBAHS, Accra, Ghana

${ }^{7}$ Biochemistry, Kwame Nkrumah University of Science and Technology, Kumasi, Ghana

${ }^{8}$ Department of Medical Biochemistry, University of Namibia School of Medicine, Windhoek, Namibia

*Corresponding author: Ababio GK, Department of Medical Biochemistry, School of Biomedical and Allied Health Sciences, University of Ghana, P. O. Box 143, Accra, Ghana, Tel: +233 20 8182253; E-mail: graceababio2002@yahoo.com

Received date: June 20, 2017; Accepted date: July 08, 2017; Published date: July 16, 2017

Copyright: ( 2017 Ababio GK, et al. This is an open-access article distributed under the terms of the Creative Commons Attribution License, which permits unrestricted use, distribution, and reproduction in any medium, provided the original author and source are credited.

\begin{abstract}
Background: Lactate dehydrogenase $(\mathrm{LDH})$ is a multifaceted enzyme whose effects in pregnancy related complications e.g., preeclampsia (PE) is now gaining attention. Here we present evidence that LDH levels could contribute significantly to the outcomes of PE.
\end{abstract}

Aim: To determine the effects of LDH in PE.

Methodology: The case control study was located at the Obstetrics and Gynaecology department of the KorleBu Teaching Hospital (KBTH). STROBE consensus checklist was adopted. One hundred and forty (140) consented subjects were recruited after ethical clearance was obtained and structured questionnaire administered to them. Four (4) $\mathrm{mL}$ blood and $5 \mathrm{~mL}$ urine samples were taken for biochemical analysis and urinalysis respectively. Randox and Sysmex automated chemistry analyser was used to quantify blood chemistry. The data was captured as protected health information (PHI) and analysed with SPSS version 22.

Results: LDH exposure was associated with higher odds of outcome in preeclampsia (PE) $[\mathrm{OR}(\mathrm{Cl})=4.76(1.26-18.72)$; $\mathrm{p}$-value $=0.0068]$. However, with an adjusted OR, LDH categories were associated with birth weight. Notwithstanding the added input, in preeclampsia, increased LDH at $<34$ weeks of gestation related with decreased birthweight only when platelet, diastolic blood pressure (DBP), $\mathrm{pH}$, bilirubin, parity and liver enzymes each served as covariates in the log linear logit analysis.

Conclusion: $\mathrm{LDH}$ was associated with low birth weight in PE in a concentration dependent manner under the influence of predictors like $\mathrm{pH}$, platelet and diastolic pressure (DBP) for causality. Therefore, a thoughtful planned foetal delivery under a specific $\mathrm{LDH}$ threshold and a regular monitoring of urine $\mathrm{pH}$, full blood count (FBC), and blood pressure might improve the outcomes of $\mathrm{PE}$

Keywords: LDH; Preeclampsia; Endothelial activation

\section{Introduction}

Lactate dehydrogenase (LDH) is a multifaceted enzyme with five (5) isozymes, all of which could occur in the placenta; but its effects in pregnancy related complications e.g., preeclampsia (PE) is now gaining attention [1,2]. Following the different kinds of treatment (e.g., expectant management and placenta delivery) of $\mathrm{PE}$ that has challenged clinical regimens, it seems expedient to find ways of preventing PE. LDH may provide such platform of data support, since increased LDH levels had been known to upregulate vascular endothelial growth factor (VEGF-A) and indirectly induce basic fibroblast growth factor (bFGF), a critical component of embryonic stem cell [3-6]. Thus, increased levels of LDH in PE is of public health concern. Reference range for $\mathrm{LDH}$ level in humans is $\leq 248 \mathrm{U} / \mathrm{L}$.
The major stimulants for $\mathrm{LDH}$ and its product, lactate, are $\mathrm{pH}$ and hypoxia. Hypoxia, when encountered in preeclampsia, increases glycolytic rate thereby increasing the activity of LDH which catalyses' the reversible reaction of pyruvate to lactate [3-13]. This reaction largely occurs in anaerobic glycolysis (or hypoxic conditions) indicating fatigue in normal persons as lactate accumulates. During fatigue or after strenuous exercise, serum proteins (e.g., LDH, aspartate aminotransferase, alanine amino transferase, albumin, and creatinine) have also been reported to change. In extreme cases or disease situations, cell death ensues as leakage of LDH outside of the cell occurs.

We therefore envisaged that under strenuous conditions e.g., pregnancy, hypoxia in preeclampsia or if body's $\mathrm{pH}$ is high; LDH production would be triggered and contribute significantly to the outcomes of PE, hence the focus. 


\section{Materials and Methods}

\section{Aim}

To determine the effects of $\mathrm{LDH}$ in $\mathrm{PE}$.

\section{Method}

This study was part of a bigger research of which one another article was duly published [14].

For the purposes of this study, PE was defined based on American College of Obstetricians and Gynaecologist criteria. Accordingly, PE is defined as diastolic blood pressure of $\geq 90 \mathrm{mmHg}$ and or systolic blood pressure of $\geq 140 \mathrm{mmHg}$ with proteinuria $\geq 300 \mathrm{mg} / \mathrm{dL}$ occurring after 20 weeks of gestation.

\section{Inclusion and exclusion criteria}

Only patients diagnosed with PE were recruited after obtaining their consent as cases. Control subjects included normotensive pregnant and non-pregnant women who gave informed consent Patients with a history of renal disease, chronic hypertension, diabetes, molar pregnancy, urinary tract infection, thyroid dysfunction and infectious diseases were excluded.

\section{Sample size}

The minimum sample size was determined using the formula in Figure 1:

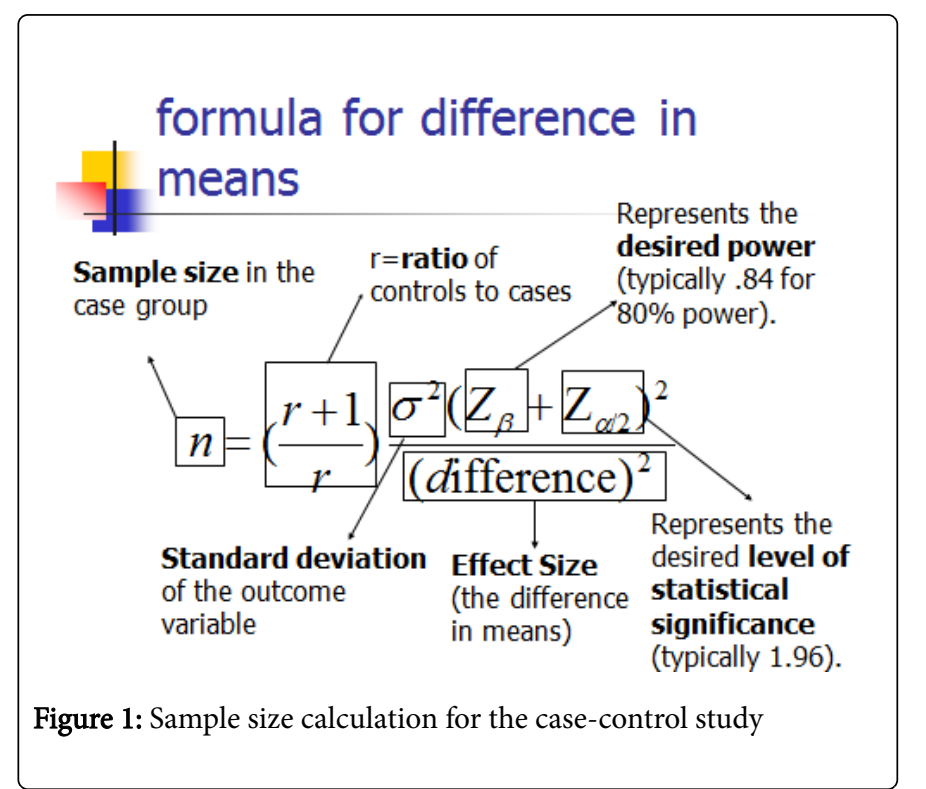

$\mathrm{Z}_{\alpha / 2}=$ standard score for the confidence interval of $95 \%$ and equals 1.96 .

$\mathrm{r}=1$ if the ratio of controls to cases were the same

Difference in means $=1.5 ; \alpha=6.27$; then $n=34.94$ samples in the case group

A $10 \%$ factor of lost to follow-up was included (3.5 samples); thereby making a total sample size of 38.44 and this was rounded up as 38. Also, all other interacting factors and priori confounding variables were duly controlled by stratification.

\section{Anthropometry}

A questionnaire for clinical information was obtained after informed consent and ethical review (MS-Et/M.3-P.3.2/2013-2014) for this case-control study situated at the Obstetrics and Gynaecology unit, Korle-Bu teaching hospital, Accra. Korle-Bu teaching hospital is one of the biggest referral centre in Ghana, located in the capital city of the country. One hundred and seventy (170) participants showed interest in the study. However, one hundred and forty (140) participants made up of sixty-four (64) controls forty-one (41) pregnant normotensives and thirty-five (35) PE; completed the study. Only patients diagnosed with PE were recruited after obtaining their consent as cases. Multiple control subjects included were normotensive pregnant and non-pregnant women, after informed consent. Multiple controls were included because patients who came to the hospital were not representative of the population, so to avoid selection bias, patients from the same hospital who apparently do not have the outcome of interest and were normal individuals with no pregnancy were selected. Also, hospitalized patients were likely to suffer health problems hence, the alternate approach was to enrol community controls i.e., individuals with no pregnancy.

\section{Dependent variable}

\section{LDH levels.}

\section{Independent variables}

Urine $\mathrm{pH}$, body mass index (BMI), age, aspartate aminotransferase, alanine amino transferase, albumin, blood pressure, creatinine, lactate dehydrogenase, week of gestation and full blood count (FBC).

\section{Blood collection and processing}

Four millilitre $(4 \mathrm{~mL})$ blood was drawn from an antecubital vein by means of a plastic syringe and dispensed into EDTA (for FBC) and gel tubes taking careful precautions. Sysmex haematological auto analyser was used to quantify FBC whilst RANDOX auto analyser was used for the determination of albumin, bilirubin, $\mathrm{LDH}$ and creatinine levels.

\section{LDH activity determination (principle)}

The LDH method measured the oxidation of L-lactate to pyruvate with simultaneous reduction of nicotinamide adenine dinucleotide (NAD). The change in absorbance at $340 \mathrm{~nm}$ due to the appearance of reduced NAD (NADH) was directly proportional to the LDH activity, since other reactants were present in non-rate limiting quantities and was measured using a biochromatic $(340,383 \mathrm{~nm})$ rate technique.

\section{Urinalysis}

Five $\mathrm{mL}$ spot urine was obtained to determine proteinuria and to categorize subjects into normotensives and PE.

\section{Data management}

Data were created on a spreadsheet, corrected for errors, three (3) different backups created and SPSS version 22 used for analysis.

\section{Statistical analysis}

Weights and heights were measured and computed for body mass index (BMI). Analysis of variance (ANOVA) was used to test for 
Citation: $\quad$ Ababio GK, Adu-Bonsaffoh K, Narh G, Morvey D, Botchway F, et al. (2017) Effects of Lactate Dehydrogenase (LDH) in Preeclampsia. .

Page 3 of 6

differences in clinical parameters between PE and the unrelated normotensives. Statistical findings from ANOVA were subjected to Ttest analysis to determine where the differences occurred. Bivariate analysis was also used to determine empirical relationship between LDH and other study variables. Logit Linear regression model was used to investigate if there was statistically significant relationship among $\mathrm{LDH}$, platelets, blood pressure, $\mathrm{pH}$ and outcomes of pregnancy. Odds ratio (OR) and confidence interval (CI) was computed to show the measure of association that existed between LDH exposure and outcome. The analyses were performed using SPSS version 22.

\section{Results and Discussion}

Out of a hundred and seventy (170) participants, only 140 subjects completed the study. The frequency of maternal indicators and pregnancy outcome indicators of PE were shown in Table 1. Even though the ages and BMI of participants were apparently similar, BMI inferential statistics were not statistically significant. Age, blood pressure, liver enzymes, albumin, platelets and creatinine therefore showed strong evidence against the null hypothesis (Table 2). The mean LDH levels in PE, pregnant normotensive and non -pregnant normotensive were $312.77 \pm 158.64(47) ; 221.29 \pm 76.17(49)$ and 356.75 $\pm 108.86(47)$ respectively.

\begin{tabular}{|c|c|c|}
\hline Parameter & $\begin{array}{l}\text { Cases with LDH }<600 \\
\text { U/L }\end{array}$ & $\begin{array}{l}\text { Cases with LDH } 600 \text { - } \\
800 \mathrm{U} / \mathrm{L}\end{array}$ \\
\hline Induction & $N=7$ & $\mathrm{~N}=1$ \\
\hline Yes & $5(71.4)$ & 1 \\
\hline No & $2(28.6)$ & \\
\hline $\mathrm{C} / \mathrm{S}$ & $N=3$ & $\mathrm{~N}=1$ \\
\hline Yes & $2(66.7)$ & 1 \\
\hline No & 1(33.3) & \\
\hline Eclampsia & $N=4$ & $\mathrm{~N}=2$ \\
\hline Yes & $2(50)$ & $1(50)$ \\
\hline No & $2(50)$ & $1(50)$ \\
\hline Laparotomy & $N=5$ & $\mathrm{~N}=1$ \\
\hline Yes & $1(20)$ & $0(0)$ \\
\hline No & $4(80)$ & $1(100)$ \\
\hline Coagulation & $N=7$ & $\mathrm{~N}=2$ \\
\hline Yes & $0(0)$ & $1(50)$ \\
\hline No & $7(100)$ & $1(50)$ \\
\hline Transfusion & $N=6$ & $\mathrm{~N}=2$ \\
\hline Yes & $0(0)$ & $2(100)$ \\
\hline No & $6(100)$ & $0(0)$ \\
\hline Respiratory distress & $\mathrm{N}=6$ & $\mathrm{~N}=1$ \\
\hline Yes & $0(0)$ & $0(0)$ \\
\hline No & $6(100)$ & $1(100)$ \\
\hline Intubation & $\mathrm{N}=5$ & $\mathrm{~N}=1$ \\
\hline
\end{tabular}

\begin{tabular}{|l|l|l|}
\hline Yes & $1(20)$ & $0(0)$ \\
\hline No & $4(80)$ & $1(100)$ \\
\hline Still birth & $\mathrm{N}=6$ & $\mathrm{~N}=1$ \\
\hline Yes & $3(50)$ & $1(100)$ \\
\hline No & $3(50)$ & $0(0)$ \\
\hline IUGR & $\mathrm{N}=4$ & \\
\hline Yes & $1(25)$ & \\
\hline No & $3(75)$ & \\
\hline
\end{tabular}

Table 1: Maternal and pregnancy outcome indicators in PE according to LDH level.

\begin{tabular}{|c|c|c|c|c|c|c|c|}
\hline & $\begin{array}{l}\text { Pregnan } \\
\text { t } \\
\text { normote } \\
\text { nsive }\end{array}$ & $\begin{array}{l}\text { Control } \\
\text { (ctrl) }\end{array}$ & $\begin{array}{l}\text { Preecla } \\
\text { mpsia }\end{array}$ & $\begin{array}{l}\text { ANOV } \\
\text { A } \\
\text { P- } \\
\text { value }\end{array}$ & $\begin{array}{l}\text { "Preg. } \\
\text { Norm } \\
0 \quad x \\
\text { Ctrl }\end{array}$ & $\begin{array}{ll}\text { PE } & X \\
\text { ctrl } & \end{array}$ & $\begin{array}{l}\text { PE } \quad x \\
\text { prg.nor } \\
\text { mo }\end{array}$ \\
\hline $\begin{array}{l}\text { Age } \\
\text { (years) }\end{array}$ & $\begin{array}{l}30.29 \pm \\
5.25(41)\end{array}$ & $\begin{array}{l}38.38 \quad \pm \\
11.00 \\
(64)\end{array}$ & $\begin{array}{l}31.00 \pm \\
5.89 \\
(35)\end{array}$ & 0 & $\begin{array}{l}0.000 \\
1\end{array}$ & 0.0004 & 0.5802 \\
\hline $\begin{array}{l}\text { BMl } \\
\left(\mathrm{kg} / \mathrm{m}^{2}\right)\end{array}$ & $\begin{array}{l}29.13 \pm \\
5.18(37)\end{array}$ & $\begin{array}{l}27.89 \pm \\
6.47(48)\end{array}$ & $\begin{array}{l}29.32 \pm \\
6.84 \\
(25)\end{array}$ & 0.537 & $\begin{array}{l}0.343 \\
1\end{array}$ & 0.3825 & 0.9014 \\
\hline $\begin{array}{l}\text { SBP } \\
(\mathrm{mmHg})\end{array}$ & $\begin{array}{l}110.05 \pm \\
14.54 \\
(41)\end{array}$ & $\begin{array}{l}108.40 \pm \\
20.43 \\
(41)\end{array}$ & $\begin{array}{l}144.63 \\
\pm 30.22 \\
(33)\end{array}$ & 0.043 & $\begin{array}{l}0.674 \\
6\end{array}$ & 0.0001 & 0.0001 \\
\hline $\begin{array}{l}\text { DBP } \\
(\mathrm{mmHg})\end{array}$ & $\begin{array}{l}71.12 \quad \pm \\
10.74 \\
(41)\end{array}$ & $\begin{array}{l}70.61 \\
12.61 \\
(41)\end{array}$ & $\begin{array}{l}98.92 \pm \\
24.41 \\
(33)\end{array}$ & 0 & 0.844 & 0.0001 & 0.0001 \\
\hline Alanine & $\begin{array}{l}6.14 \pm \\
3.45(52)\end{array}$ & $\begin{array}{l}15.65 \pm \\
4.34(39)\end{array}$ & $\begin{array}{l}10.41 \pm \\
10.30 \\
(42)\end{array}$ & 0 & $\begin{array}{l}0.000 \\
1\end{array}$ & 0.0043 & 0.0062 \\
\hline \multicolumn{8}{|l|}{$\begin{array}{l}\text { aminotr } \\
\text { ansfera } \\
\text { se }(U / L)\end{array}$} \\
\hline $\begin{array}{l}\text { Asparta } \\
\text { te }\end{array}$ & $\begin{array}{l}13.04 \pm \\
3.91(52)\end{array}$ & $\begin{array}{l}27.42 \quad \pm \\
14.34 \\
(39)\end{array}$ & $\begin{array}{l}34.45 \pm \\
20.39 \\
(43)\end{array}$ & 0 & $\begin{array}{l}0.000 \\
1\end{array}$ & 0.0775 & 0.0001 \\
\hline \multicolumn{8}{|l|}{$\begin{array}{l}\text { aminotr } \\
\text { ansfera } \\
\text { se }(U / L)\end{array}$} \\
\hline Urea & $\begin{array}{l}2.65 \pm \\
0.66(52)\end{array}$ & $\begin{array}{l}2.97 \quad \pm \\
0.44(64)\end{array}$ & $\begin{array}{l}3.17 \\
1.05 \\
(43)\end{array}$ & 0.002 & $\begin{array}{l}0.002 \\
3\end{array}$ & 0.1771 & 0.0042 \\
\hline $\begin{array}{l}\text { Urine } \\
\mathrm{pH}\end{array}$ & $\begin{array}{l}6.01 \pm \\
0.97(27)\end{array}$ & $\begin{array}{l}5.37 \pm \\
0.76(46)\end{array}$ & $\begin{array}{l}5.89 \pm \\
0.76(19 \\
)\end{array}$ & $\begin{array}{l}0.000 \\
4\end{array}$ & $\begin{array}{l}0.014 \\
7\end{array}$ & 0.0025 & 0.6548 \\
\hline Albumin & $\begin{array}{l}32.98 \quad \pm \\
3.10(52)\end{array}$ & $\begin{array}{l}40.43 \pm \\
6.89(64)\end{array}$ & $\begin{array}{l}30.04 \pm \\
4.31 \\
(44)\end{array}$ & 0 & $\begin{array}{l}0.000 \\
1\end{array}$ & 0.0001 & 0.0002 \\
\hline $\begin{array}{l}\text { Bilirubin } \\
\text { total }\end{array}$ & $\begin{array}{l}11.08 \pm \\
8.49(52)\end{array}$ & $\begin{array}{l}10.97 \pm \\
7.95(61)\end{array}$ & $\begin{array}{l}7.48 \\
3.71 \\
(44)\end{array}$ & 0 & $\begin{array}{l}0.943 \\
5\end{array}$ & 0.008 & 0.0106 \\
\hline
\end{tabular}


Citation: $\quad$ Ababio GK, Adu-Bonsaffoh K, Narh G, Morvey D, Botchway F, et al. (2017) Effects of Lactate Dehydrogenase (LDH) in Preeclampsia. .

Page 4 of 6

\begin{tabular}{|c|c|c|c|c|c|c|c|}
\hline $\begin{array}{l}\text { Bilirubin } \\
\text { (direct) }\end{array}$ & $\begin{array}{l}7.78 \quad \pm \\
5.11(52)\end{array}$ & $\begin{array}{l}8.55 \\
7.2(61)\end{array}$ & $\begin{array}{ll}4.49 & \pm \\
1.69 & \\
(44) & \end{array}$ & 0.001 & $\begin{array}{l}0.520 \\
3\end{array}$ & 0.0004 & 0.0001 \\
\hline $\begin{array}{l}\text { Bilirubin } \\
\text { (uncon. } \\
\text { ) }\end{array}$ & $\begin{array}{l}3.31 \quad \pm \\
7.33(52)\end{array}$ & $\begin{array}{l}2.42 \pm \\
2.81(61)\end{array}$ & $\begin{array}{ll}2.99 & \pm \\
3.01 & \\
(44) & \end{array}$ & 0.612 & $\begin{array}{l}0.382 \\
8\end{array}$ & 0.3219 & 0.7872 \\
\hline $\begin{array}{l}\text { PLT } \\
(\times 109 / L \\
)\end{array}$ & $\begin{array}{l}241.17 \quad \pm \\
75.62 \\
(48)\end{array}$ & $\begin{array}{l}299.46 \pm \\
85.43 \\
(48)\end{array}$ & $\begin{array}{l}232.10 \\
\pm 92.09 \\
(31)\end{array}$ & 0 & $\begin{array}{l}0.000 \\
6\end{array}$ & 0.0014 & 0.6343 \\
\hline Hct & $\begin{array}{l}0.33 \quad \pm \\
0.04(48)\end{array}$ & $\begin{array}{l}0.39 \quad \pm \\
0.06(48)\end{array}$ & $\begin{array}{ll}0.38 & \pm \\
0.04 & \\
(28) & \end{array}$ & 0 & 0 & 0.435 & 0 \\
\hline $\begin{array}{l}\text { Creatini } \\
\text { ne } \\
\text { (umol/L } \\
\text { ) }\end{array}$ & $\begin{array}{l}51.64 \quad \pm \\
13.99 \\
(52)\end{array}$ & $\begin{array}{ll}68.43 & \pm \\
11.28 & \\
(48) & \end{array}$ & $\begin{array}{l}63.19 \pm \\
20.06 \\
(43)\end{array}$ & 0 & $\begin{array}{l}0.000 \\
1\end{array}$ & 0.1232 & 0.0014 \\
\hline $\begin{array}{l}\text { Weeks } \\
\text { of } \\
\text { gestatio } \\
\mathrm{n}\end{array}$ & $\begin{array}{l}30.98 \quad \pm \\
6.42(41)\end{array}$ & & $\begin{array}{l}32.46 \pm \\
5.89 \\
(24)\end{array}$ & 0.359 & & & 0.359 \\
\hline $\begin{array}{l}\text { Number } \\
\text { of prev. } \\
\text { births }\end{array}$ & $\begin{array}{l}1.84 \pm \\
1.28(25)\end{array}$ & & $\begin{array}{ll}2.60 & \pm \\
1.42 & \\
(20) & \end{array}$ & 0.066 & & & 0.066 \\
\hline $\begin{array}{l}\text { Number } \\
\text { of preg. }\end{array}$ & $\begin{array}{l}2.43 \pm \\
1.52(40)\end{array}$ & & $\begin{array}{ll}3.26 & \pm \\
1.83 & \\
(29) & \end{array}$ & 0.044 & & & 0.044 \\
\hline $\begin{array}{l}\text { Number } \\
\text { of } \\
\text { antenat } \\
\text { al visits }\end{array}$ & $\begin{array}{l}4.35 \quad \pm \\
2.82(34)\end{array}$ & & $\begin{array}{ll}5.00 & \pm \\
2.21 & \\
(28) & \end{array}$ & 0.324 & & & 0.324 \\
\hline $\begin{array}{l}\text { Birth } \\
\text { weight }\end{array}$ & $\begin{array}{l}2687.71 \\
\pm \quad 845.05 \\
(24)\end{array}$ & & $\begin{array}{l}1608.75 \\
\pm \\
1132.90 \\
(12)\end{array}$ & $\begin{array}{l}0.002 \\
8\end{array}$ & & & 0.0028 \\
\hline
\end{tabular}

\begin{tabular}{|c|c|c|c|c|c|c|c|c|c|}
\hline \multirow{2}{*}{\multicolumn{2}{|c|}{ IDCat1a }} & \multirow[b]{2}{*}{ B } & \multirow[b]{2}{*}{ S.E. } & \multirow[b]{2}{*}{ Wald } & \multirow[b]{2}{*}{ df } & \multirow[b]{2}{*}{ Sig. } & \multirow{2}{*}{$\begin{array}{l}\operatorname{Exp}( \\
\text { B) }\end{array}$} & \multicolumn{2}{|c|}{$\begin{array}{l}\text { 95\% Confidence } \\
\text { Interval }\end{array}$} \\
\hline & & & & & & & & Lower & Upper \\
\hline $\begin{array}{l}\text { Step } \\
0^{\text {a }}\end{array}$ & $\begin{array}{l}\text { Con } \\
\text { stant }\end{array}$ & -1.299 & 0.376 & 11.938 & 1 & $\begin{array}{l}0.00 \\
1\end{array}$ & $\begin{array}{l}0.27 \\
3\end{array}$ & -2.001 & -0.693 \\
\hline \multirow{2}{*}{\multicolumn{2}{|c|}{ IDCat2a }} & \multirow[b]{2}{*}{ B } & \multirow[b]{2}{*}{ S.E. } & \multirow[b]{2}{*}{ Wald } & \multirow[b]{2}{*}{ df } & \multirow[b]{2}{*}{ Sig. } & \multirow{2}{*}{$\begin{array}{l}\text { Exp( } \\
\text { B) }\end{array}$} & \multicolumn{2}{|c|}{$\begin{array}{l}\text { 95\% Confidenc } \\
\text { Interval }\end{array}$} \\
\hline & & & & & & & & Lower & Upper \\
\hline $\begin{array}{l}\text { Step } \\
0^{\mathrm{a}}\end{array}$ & $\begin{array}{l}\text { Con } \\
\text { stant }\end{array}$ & 0.036 & 0.27 & 0.018 & 1 & $\begin{array}{l}0.89 \\
3\end{array}$ & $\begin{array}{l}1.03 \\
7\end{array}$ & -2.001 & -0.693 \\
\hline
\end{tabular}

a. Variable(s) entered in step 1: BMI5, sbp10, BMlcat, SBPcat, birth weight, IDCat1a or IDCat2a.

b. BMIcat=BMI categories; SBPcat=SBP categories; BMI5=BMI increment of 5 ; $\operatorname{Exp}(B)=$ Odds ratio; S.E. =standard error; df=degree of freedom; IDCat1 $a=I D$ categories e.g., PE and pregnant Normotensive only; IDCat2a=ID categories

e.g., PE and non-pregnant Normotensive only

Table 4: Adjusted Odds ratio using IDCat1a and IDCat2a.

"T-test was performed to show the parameters influencing the ANOVA statistical significance. $\mathrm{SBP}=$ systolic blood pressure, $\mathrm{DBP}=$ diastolic blood pressure, $\mathrm{Hct}=$ hematocrit, Plt=platelet, $\mathrm{BMI}=$ body mass index

Table 2: Clinical variables of subjects at $\mathrm{LDH}<600 \mathrm{U} / \mathrm{L}$.

LDH activity was seen to be associated with higher odds of outcome in $\mathrm{PE}[\mathrm{OR}(\mathrm{CI})=4.76(1.26-18.72)$; $\mathrm{p}$-value $=0.0068]$. With an adjusted OR from binary logistics (Tables 3-6), confounding variables controlled and adjustment made with SBP increment of 10 plus BMI increment of 5, $\mathrm{LDH}$ categories were seen to be associated with birth weight.

\begin{tabular}{|c|c|c|c|}
\hline \multicolumn{3}{|l|}{ LDH Levels } & \\
\hline & Exposed & Unexposed & \multirow{3}{*}{$\begin{array}{l}\text { OR }(\mathrm{Cl})=4.76(1.26-18.72) \\
\text { Chi-square }=7.33 \\
\text { p-value }=0.0068\end{array}$} \\
\hline Cases (PE) & 10 & 14 & \\
\hline $\begin{array}{l}\text { Controls } \\
\text { (Pregnant } \\
\text { Normotensive) }\end{array}$ & 6 & 40 & \\
\hline
\end{tabular}

Table 3: Odds ratio (OR) showing the risk of elevated LDH levels in the population.

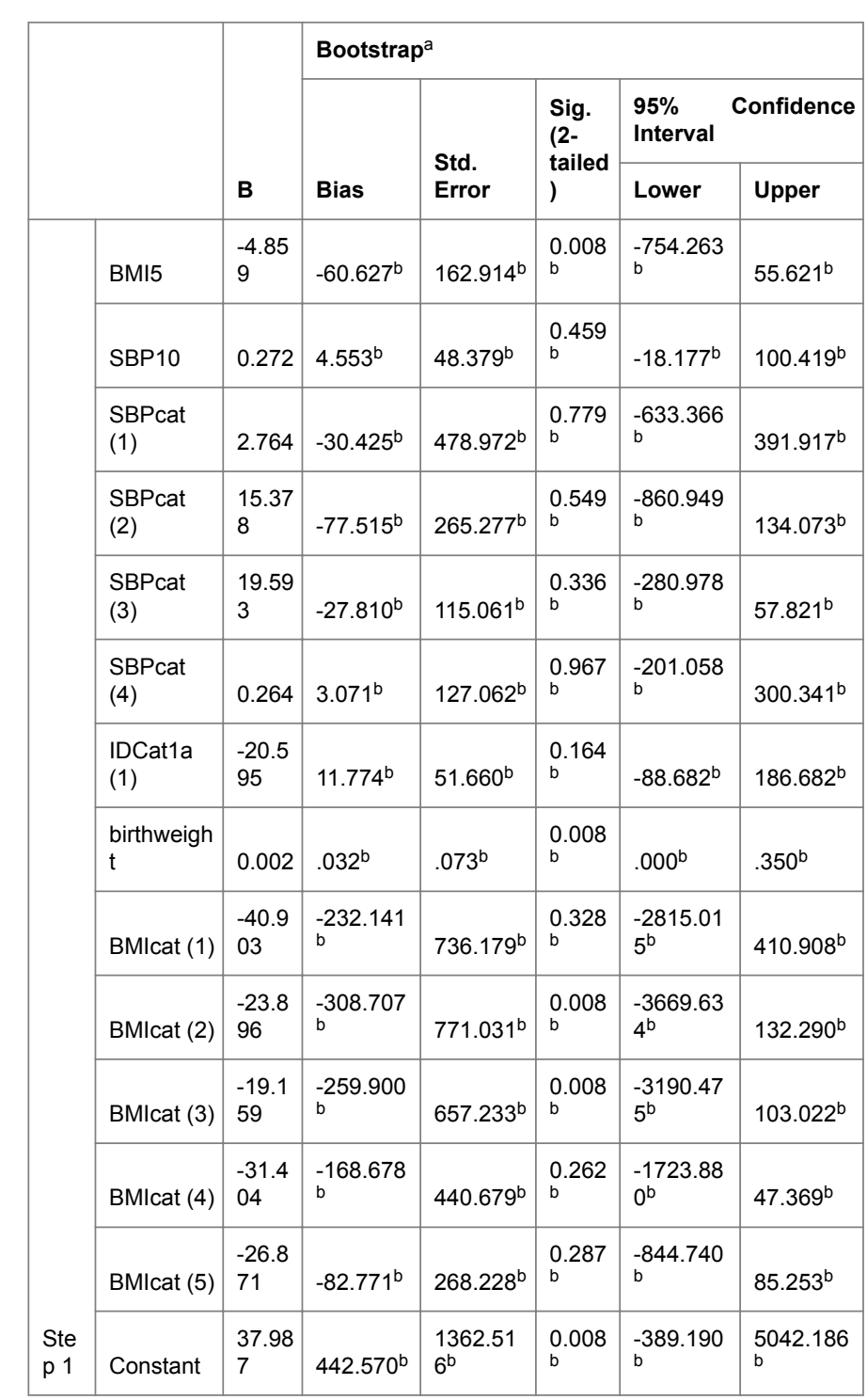


Page 5 of 6

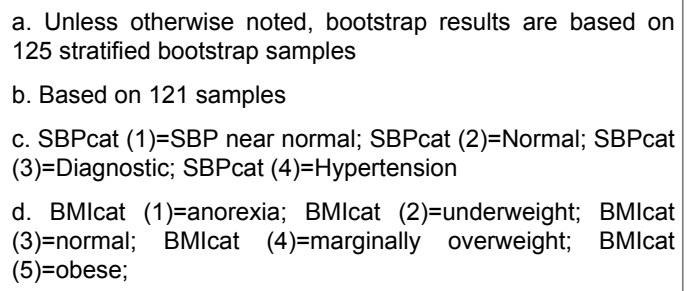

Table 5: Bootstrap for Variables in the Equation (using IDCat1a): both SBP and BMI adjusted.

\begin{tabular}{|c|c|c|c|c|c|c|c|}
\hline & & \multirow[b]{3}{*}{ B } & \multicolumn{5}{|c|}{ Bootstrapa } \\
\hline & & & \multirow[b]{2}{*}{ Bias } & \multirow{2}{*}{$\begin{array}{l}\text { Std. } \\
\text { Error }\end{array}$} & \multirow{2}{*}{$\begin{array}{l}\text { Sig. (2- } \\
\text { tailed) }\end{array}$} & \multicolumn{2}{|c|}{$\begin{array}{l}95 \% \text { Confidence } \\
\text { Interval }\end{array}$} \\
\hline & & & & & & Lower & Upper \\
\hline & BMI5 & -0.422 & 0.388 & 1.137 & 0.627 & -2.502 & 2.409 \\
\hline & sbp10 & 0.27 & 0.061 & 0.324 & 0.238 & -0.338 & 0.989 \\
\hline & SBPcat (1) & 0.885 & 4.631 & 11.244 & 0.381 & -21.236 & 23.803 \\
\hline & SBPcat (2) & -0.221 & -0.982 & 4.635 & 0.794 & -19.626 & 2.807 \\
\hline & SBPcat (3) & -0.102 & -0.512 & 6.125 & 0.865 & -19.679 & 20.942 \\
\hline & SBPcat (4) & -2.284 & -1.178 & 4.422 & 0.008 & -21.474 & -0.42 \\
\hline & birthweight & 0.001 & 0 & 0.001 & 0.016 & 0 & 0.005 \\
\hline & BMIcat (1) & $\begin{array}{l}18.34 \\
8\end{array}$ & 2.137 & 6.216 & 0.032 & 6.28 & 36.277 \\
\hline & BMlcat (2) & -2.976 & 1.703 & 5.391 & 0.437 & -14.244 & 10.772 \\
\hline & BMlcat (3) & -2.071 & 1.561 & 4.261 & 0.556 & -9.952 & 10.247 \\
\hline & BMIcat (4) & -2.784 & 1.022 & 3.329 & 0.262 & -8.896 & 5.831 \\
\hline & BMlcat (5) & $\begin{array}{l}20.30 \\
6\end{array}$ & 0.784 & 2.25 & 0.008 & 17.188 & 27.329 \\
\hline \multirow{2}{*}{$\begin{array}{l}\text { Step } \\
1\end{array}$} & IDcat2a (1) & 4.782 & 2.51 & 5.119 & 0.008 & 3.618 & 25.621 \\
\hline & Constant & -2.928 & -5.772 & 12.099 & 0.762 & -37.728 & 15.284 \\
\hline
\end{tabular}

Table 6: Bootstrap for Variables in the Equation (using IDCat2a): both SBP and BMI adjusted.

Inferences from logit showed increased LDH (at a lesser week of gestation) relating with decreased birthweight under the influence of platelet, DBP, $\mathrm{pH}$, bilirubin, parity and liver enzymes which duly served as covariates. The bivariate analysis indicated a significant correlation of LDH with SBP, DBP, creatinine, liver enzymes (ALT and AST), total bilirubin, direct bilirubin, unconjugated bilirubin and urine $\mathrm{pH}$, whilst pregnancy outcome (birth weight) correlated with DBP, total bilirubin, and direct bilirubin. Wherever DBP, total bilirubin, and direct bilirubin correlated with birthweight, these parameters were also found to correlate with LDH. Other notable bivariate correlation(s) were: age of subjects correlating with platelets; liver enzymes correlating with SBP and LDH; weeks of gestation correlating with creatinine, haematocrit and urine $\mathrm{pH}$; $\mathrm{BMI}$ correlating with direct bilirubin; bilirubin (total, conjugated or unconjugated) correlating with weeks of gestation; and creatinine correlating with urea, $\mathrm{LDH}$ and urine $\mathrm{pH}$.

In this study, a report on the effects of LDH on the outcomes of PE were presented. Among preeclampsia, the relationship between LDH and birth weight in the current study was concentration dependent only when notable factors like $\mathrm{pH}$, platelet and diastolic blood pressure (DBP) served as predictors. Even though there were several factors that trigger $\mathrm{LDH}$, we are by this study adding on to literature that increased $\mathrm{pH}$, decreased platelet and increased DBP found in this study could possibly trigger LDH production in PE massively [3,4,9-11,13].

Indeed, the current study provided information for the first time in Ghana on LDH levels in PE and how adjusted odds ratio with SBP increments of ten (10) and BMI increment of five (5) could make LDH closely relate with birth weight. To the best of our knowledge, this is the first study of a kind in the country to elucidate the effects of LDH on the outcomes of PE.

In $\mathrm{PE}$, an intense systemic inflammatory response associated with changes in hemodynamics were observed. In contrast, a milder trend was duly observed in normal pregnancy. However, it did not indicate illness except for the changes in hemodynamics [15]. As pregnancy advanced, the systemic inflammatory response strengthened, reaching a peak at the third trimester. Therefore, the systemic inflammatory response combined with systemic endothelial dysfunction which became much more pronounced in PE $[16,17]$.

The incidence of PE complications recorded might have been influenced by our study size, socioeconomic status and hemodynamic changes. The alternate approach in enrolling non - pregnant normotensive duly compensated for almost all the blood chemistry with the exception of a few outliers in LDH levels.

Also, a good anticipation from literature points to reducing equivalents (e.g., NADH) formed in the cell during the reaction of lactate to pyruvate under the influence of $\mathrm{LDH}$. NADH, by this process are taken up by malate dehydrogenase in the malate-aspartate shuttle $[18,19]$. This could possibly explain why the liver enzymes especially AST levels increased in PE.

\section{Conclusion}

The relationship between $\mathrm{LDH}$ and birth weight in PE seemed to be concentration dependent which needed predictors like $\mathrm{pH}$, platelet and diastolic pressure (DBP) for causality. Therefore, a routine monitoring of $\mathrm{LDH}$ levels, urine $\mathrm{pH}, \mathrm{FBC}$ and blood pressure in $\mathrm{PE}$ as well as a planned foetal delivery under a specific LDH threshold might improve the outcomes of PE.

\section{Limitation}

Selection of a suitable control was a major challenge since those from the hospital setting had outcomes related to the exposure being studied, hence two control(s) e.g., pregnant normotensive and nonpregnant normotensive per case were recruited to improve the statistical power of the study.

\section{Acknowledgement}

This work was supported by grants from ORID, University of Ghana, Legon. The authors duly acknowledge Mensah J, Amponsah C, 
Citation: $\quad$ Ababio GK, Adu-Bonsaffoh K, Narh G, Morvey D, Botchway F, et al. (2017) Effects of Lactate Dehydrogenase (LDH) in Preeclampsia. .

Page 6 of 6

staff of Obstetrics and Gynaecology of the Korle-Bu Teaching Hospital, Accra, Ghana; and, Medical Biochemistry, Univ. of Ghana SBAHS.

\section{Funding Disclosure}

Work from the authors' laboratory was supported by grants from ORID, University of Ghana, Legon.

\section{Conflict of Interest}

The authors declare that there is no conflict of interest.

\section{References}

1. Kim JW, Dang CV (2005) Multifaceted roles of glycolytic enzymes. Trends Biochem Sci 30: 142-150.

2. Tsoi SC, Zheng J, Xu F, Kay HH (2001) Differential Expression of Lactate Dehydrogenase Isozymes (LDH) in Human Placenta with High Expression of LDH-A 4 Isozyme in the Endothelial Cells of Pre-eclampsia Villi. Placenta 22: 317-322

3. Semenza GL (2003) Targeting HIF-1 for cancer therapy. Nat Rev Cancer 3: 721-732.

4. Semenza GL (2010) HIF-1: upstream and downstream of cancer metabolism. Curr Opin Genet Dev 20: 51-56.

5. Pepper MS, Belin D, Montesano R, Orci L, Vassalli JD (1990) Transforming growth factor-13-1 modulates basic fibroblast growth factor induced proteolytic and angiogenic properties of endothelial cells in vitro. J Cell Biol 111: 743-755.

6. Mignatti P, Mazzieri R, Rifkin DB (1991) Expression of the urokinase receptor in vascular endothelial cells is stimulated by basic fibroblast growth factor. J Cell Biol 113: 1193-1201.

7. Davies DD, Grego S, Kenworthy P (1974) The control of the production of lactate and ethanol by higher plants. Planta 118: 297-310.
8. Granger JP, Alexander BT, Llinas MT, Bennett WA, Khalil RA (2002) Pathophysiology of preeclampsia: linking placental ischemia/hypoxia with microvascular dysfunction. Microcirculation 9: 147-160.

9. Firth JD, Ebert BL, Ratcliffe PJ (1995) Hypoxic regulation of lactate dehydrogenase a Interaction between hypoxia-inducible factor 1 and cAMP response elements. J Biol Chem 270: 21021-21027.

10. Lu H, Forbes RA, Verma A (2002) Hypoxia-inducible factor 1 activation by aerobic glycolysis implicates the Warburg effect in carcinogenesis. J Biol Chem 277: 23111-23115.

11. Lu H, Dalgard CL, Mohyeldin A, McFate T, Tait AS, et al. (2005) Reversible inactivation of HIF-1 prolyl hydroxylases allows cell metabolism to control basal HIF-1. J Biol Chem 280: 41928-41939.

12. Pugh CW, Ratcliffe PJ (2003) Regulation of angiogenesis by hypoxia: role of the HIF system. Nat Med 9: 677-684.

13. Semenza GL (2001) HIF-1 and mechanisms of hypoxia sensing. Curr Opin Cell Biol 13: 167-171.

14. Ababio GK, Adu-Bonsaffoh K, Botchway F, Abindau E, Quaye IKE (2016) Hyperuricemia and adverse pregnancy outcomes in Ghanaian women: potential mechanism. Biochem Anal Biochem 5: 275-279.

15. Peralta PML, Basavilvazo RMA, Cruz AA (2004) Clinical significance of the laboratory determinations in preeclamptic patients. Ginecol Obstet Mex 72: 57-62.

16. Var A, Yildirim Y, Onur E (2003) Endothelial dysfunction in preeclampsia: increased homocystine and decreased nitric acid levels. Gynecol Obstet Invest 56: 221-224.

17. Page NM (2002) The endocrinology of pre-eclampsia. Clin Endocrinol 57: 413-423.

18. Norwitz ER, Hsu CD, Repke JT (2002) Acute complications of preeclampsia. Clin Obstet Gynecol 45: 308-329.

19. Kane DA (2015) Lactate oxidation at the mitochondria: a lactate-malateaspartate shuttle at work. Glycolysis at 75: Is it Time to Tweak the First Elucidated Metabolic Pathway in History, p: 40. 